\title{
Beta-numbers whose conjugates lie near the unit circle
}

by

\section{DoYong Kwon (Seoul)}

1. Introduction. Let $\alpha$ and $\varrho$ be in $[0,1]$. We define two (right) infinite words $s_{\alpha, \varrho}$ and $s_{\alpha, \varrho}^{\prime}$, the $n$th terms of which are, for $n \geq 0$, given by $s_{\alpha, \varrho}(n)=\lfloor\alpha(n+1)+\varrho\rfloor-\lfloor\alpha n+\varrho\rfloor, \quad s_{\alpha, \varrho}^{\prime}(n)=\lceil\alpha(n+1)+\varrho\rceil-\lceil\alpha n+\varrho\rceil$, where $\lfloor t\rfloor$ is the largest integer not greater than $t$, and $\lceil t\rceil$ is the smallest integer not less than $t$. We see that these are infinite words over the alphabet $A_{1}=\{0,1\}$. Here $s_{\alpha, \varrho}$ (resp. $s_{\alpha, \varrho}^{\prime}$ ) is called a lower (resp. upper) mechanical word with slope $\alpha$ and intercept $\varrho$. If the slope $\alpha$ is irrational, then these infinite words are aperiodic and termed Sturmian words [13]. On the other hand, if the slope $\alpha$ is rational then they are purely periodic, and the words constituting the smallest period are called Christoffel words. For a general survey, see [11].

While Christoffel and Sturmian words have been an important subject studied in theoretical computer science, the $\beta$-transformation has been a flourishing example in ergodic theory since Rényi introduced it in [15]. Let $\beta>1$. The $\beta$-transformation $T_{\beta}: x \mapsto \beta x(\bmod 1)$ determines the $\beta$ expansion $d_{\beta}(x)$ of a given $x \in[0,1]$ by the "greedy algorithm" (except 1 ), that is to say,

$$
d_{\beta}(x):=\left(x_{i}\right)_{i \geq 1}, \quad \text { where } \quad x_{i}=\left\lfloor\beta T_{\beta}^{i-1}(x)\right\rfloor .
$$

Amongst all $\beta$-expansions of $x \in[0,1]$, the $\beta$-expansion of 1 is quite distinctive in that it is lexicographically greater than any other $\beta$-expansion of $x \in[0,1)$, and moreover this property exhaustively characterizes possible $\beta$-expansions $\sum_{i=1}^{\infty} x_{i} \beta^{i}$ of $x$ (see [14]). Now the $\beta$-shift $S_{\beta}$ is, by definition, the closure of $\left\{d_{\beta}(x) \mid x \in[0,1)\right\}$ in the full shift. Then the dynamics of $S_{\beta}$ can be addressed by regarding $d_{\beta}(1)$ from the language-theoretical point of view [14, 1]. Later, Blanchard [2] suggested a systematic study of real numbers from totally new angles; he classified real numbers $\beta>1$ into five

2000 Mathematics Subject Classification: Primary 30C15, 11R06; Secondary 37B10, $68 \mathrm{R} 15$. 
categories according to the ergodic properties of $S_{\beta}$. For language-theoretical terminology, see [2] and the references therein.

(i) $\beta \in \mathcal{C}_{1}$ if $S_{\beta}$ is a shift of finite type, or equivalently $d_{\beta}(1)$ is finite,

(ii) $\beta \in \mathcal{C}_{2}$ if $S_{\beta}$ is sofic, or equivalently $d_{\beta}(1)$ is eventually periodic,

(iii) $\beta \in \mathcal{C}_{3}$ if $S_{\beta}$ is specified,

(iv) $\beta \in \mathcal{C}_{4}$ if $S_{\beta}$ is synchronizing,

(v) $\beta \in \mathcal{C}_{5}$ if $S_{\beta}$ has none of the above properties.

So we have the following inclusions:

$$
\emptyset \neq \mathcal{C}_{1} \subset \mathcal{C}_{2} \subset \mathcal{C}_{3} \subset \mathcal{C}_{4} \subset(1, \infty), \quad \mathcal{C}_{5}=(1, \infty) \backslash \mathcal{C}_{4}
$$

A real $\beta \in \mathcal{C}_{2}$ is called a beta-number, in particular, $\beta \in \mathcal{C}_{1}$ is a simple beta-number.

In the previous papers $[8,4]$, the author showed that for any slope $\alpha>0$ there exists a unique $\beta>1$ such that $T_{\beta}$ behaves like rotation by $\alpha$ on the minimal set containing 1 . So he defined a map $\Delta: \alpha \mapsto \beta$ and also showed that $\Delta$ maps irrationals to transcendental numbers and rationals to algebraic integers. At an irrational the value of $\Delta$ is in $\mathcal{C}_{3}$ but not in $\mathcal{C}_{2}$, whereas at a rational it is some beta-number. Now our main aim is to study the algebraic properties of such beta-numbers, which are called self-Christoffel numbers. More precisely, we investigate algebraic degrees of self-Christoffel numbers over the field of rationals. This study is made possible by locating their Galois conjugates. Throughout the paper, we will just say "conjugates of $\beta$ " instead of "Galois conjugates of $\beta$ other than $\beta$ ". Closely connected with our approach is the result of Solomyak [18], and Flatto, Lagarias and Poonen [7]. They examined, in different contexts, conjugates of general beta-numbers and gave a better bound for the modulus of conjugates of beta-numbers than Parry [14] did. But in the case of self-Christoffel numbers, this bound can be substantially improved, which makes it possible to find their minimal polynomials.

2. Christoffel words and lexicographic order. We recall some definitions from language theory, which can be found in [11]. Given a finite alphabet $A$, a finite (resp. infinite) sequence of elements of $A$ is called a finite (resp. infinite) word. If it is clear from the context, we just say "word" omitting "finite" (resp. "infinite"). Let $A^{*}$ (resp. $A^{\mathbb{N}}$ ) be the set of finite (resp. infinite) words over $A$. Then $A^{*}$ is a free monoid under the concatenation operation, and the empty word $\varepsilon$ is its identity. We use the notation $A^{+}:=A^{*} \backslash\{\varepsilon\}$. Let us denote by $\sigma$ the shift of finite or infinite sequences. A word $w \in A^{*} \cup A^{\mathbb{N}}$ is said to be a factor (resp. prefix, suffix) of a word $u \in A^{*} \cup A^{\mathbb{N}}$ provided $u$ can be expressed in the form $u=x w y$ (resp. $u=w y$, $u=x w)$ for some $x$ and $y$. For an integral alphabet $A \subset \mathbb{N}$, we extend the 
usual lexicographic order on $A^{\mathbb{N}}$ to an order on $A^{*} \cup A^{\mathbb{N}}$ by substituting any $x \in A^{*}$ with $x 0^{\omega}:=x 00 \cdots$ even though $0 \notin A$. For example, if $x, y \in A^{*}$ and $z \in A^{\mathbb{N}}$, then $x<y$ (resp. $y<z$ ) if and only if $x 0^{\omega}<y 0^{\omega}$ (resp. $\left.y 0^{\omega}<z\right)$. A nonempty word $u \in A^{*}$ is primitive if $u=x^{n}$ for some $x$ implies $n=1$. For a word $u \in A^{*} \cup A^{\mathbb{N}}$, we denote by $\operatorname{alph}(u) \subset A$ the set of letters appearing in $u$, and by $F(u)$ the set of finite factors of $u$. For a subset $X \subset A^{*}$, we define $F(X):=\bigcup_{x \in X} F(x)$. We call $X$ factorial if $F(X) \subset X$. We let $|u|$ denote the length of $u$, and $|u|_{a}$ the number of times the letter $a \in A$ appears in $u$.

For convenience of exposition, we suppose $A=\{0,1\}$ and consider mechanical words with rational slope $\alpha=p / q \in(0,1) \operatorname{gcd}(p, q)=1$. These restrictions will be relaxed soon. Noting that $s_{\alpha, 0}$ and $s_{\alpha, 0}^{\prime}$ are purely periodic, we look at their minimal periods

$$
t_{p, q}=a_{0} \cdots a_{q-1}, \quad t_{p, q}^{\prime}=a_{0}^{\prime} \cdots a_{q-1}^{\prime},
$$

where

$$
a_{i}=\left\lfloor(i+1) \frac{p}{q}\right\rfloor-\left\lfloor i \frac{p}{q}\right\rfloor, \quad a_{i}^{\prime}=\left\lceil(i+1) \frac{p}{q}\right\rceil-\left\lceil i \frac{p}{q}\right\rceil .
$$

So we have $s_{\alpha, 0}=t_{p, q}^{\omega}$ and $s_{\alpha, 0}^{\prime}=t_{p, q}^{\omega}$. These words $t_{p, q}, t_{p, q}^{\prime}$ are said to be Christoffel words. One sees that $t_{1,1}=t_{1,1}^{\prime}=1$ and that they can be factored as

$$
t_{p, q}=0 z_{p, q} 1, \quad t_{p, q}^{\prime}=1 z_{p, q} 0,
$$

for some word $z_{p, q}$, called a central word. It is easy to see that Christoffel words are all primitive and that $z_{p, q}$ is a palindrome, i.e., $z_{p, q}$ is equal to its reversal. We recall here that if $\alpha$ is irrational, then $s_{\alpha, 0}=0 c_{\alpha}$ and $s_{\alpha, 0}^{\prime}=1 c_{\alpha}$ for some infinite word $c_{\alpha}$, called the characteristic word of slope $\alpha$. Conversely, if both $0 c$ and $1 c$ are Sturmian, then $c$ is known to be a characteristic word [11].

We say a subset $X \subset A^{*}$ is balanced if for any $x, y \in X,\left.|| x\right|_{1}-|y|_{1} \mid \leq 1$ whenever $|x|=|y|$. Otherwise $X$ is unbalanced. A word $u \in A^{*} \cup A^{\mathbb{N}}$ is called balanced if $F(u)$ is balanced. Coven and Hedlund [5] described the balanced property in more detail.

Proposition 2.1. Let $X$ be a factorial subset of $A^{*}$. Then $X$ is unbalanced if and only if there exists a palindrome $w$ such that both $0 w 0$ and $1 w 1$ lie in $X$.

Here are some results on finite and infinite balanced words. See [11].

Proposition 2.2.

(a) If both $0 w$ and $1 w$ are finite balanced words, then $w$ is a prefix of some characteristic word. 
(b) Let $s$ be an infinite balanced word. If $s$ is aperiodic, then $s$ is Sturmian. If $s$ is purely periodic, then $s$ is a mechanical word of rational slope.

From now on, we allow the slope to be any $\alpha \in(0, \infty)$, and adopt an alphabet $A_{b}=\{b-1, b\}$ with $b=\lceil\alpha\rceil$. Then the letters involved in the words mentioned above are substituted as $0 \mapsto b-1$ and $1 \mapsto b$, i.e.,

$$
\operatorname{alph}\left(s_{\alpha, 0}\right)=\operatorname{alph}\left(s_{\alpha, 0}^{\prime}\right)=\operatorname{alph}\left(c_{\alpha}\right)=\operatorname{alph}\left(z_{p, q}\right)=\{b-1, b\} .
$$

For instance,

- if $\alpha$ is irrational and $b=\lceil\alpha\rceil$, then $s_{\alpha, 0}=(b-1) c_{\alpha}, s_{\alpha, 0}^{\prime}=b c_{\alpha}$,

- if $b=\lceil p / q\rceil$, then $t_{p, q}=(b-1) z_{p, q} b, t_{p, q}^{\prime}=b z_{p, q}(b-1)$.

The next theorem is the main motivation of our work.

Theorem $2.3([4,8])$. There exists a function $\Delta:[0, \infty) \rightarrow[1, \infty)$ with the following propertis:

(a) If $\alpha>0$ is irrational, then the $\Delta(\alpha)$-expansion of 1 is given by $b c_{\alpha}$, where $b=\lceil\alpha\rceil$.

(b) If $\alpha=p / q$ with $p, q$ relatively prime, then the $\Delta(\alpha)$-expansion of 1 is given by $b z_{p, q} b$, where $b=\lceil\alpha\rceil$.

(c) $\Delta$ is continuous at every irrational point.

(d) At every rational point, $\Delta$ is left-continuous but not right-continuous.

(e) Given a rational $\alpha=p / q$ with $b=\lceil\alpha\rceil$, let $\beta$ be the right limit $\Delta(\alpha+):=\lim _{x \rightarrow \alpha+} \Delta(x)$. Then the $\beta$-expansion of 1 is given by $b\left(z_{p, q} b(b-1)\right)^{\omega}$.

In [14], Parry found all candidates for sequences that can be $d_{\beta}(1)$ for some $\beta>1$. Such sequences $s \in\{0,1, \ldots,\lfloor\beta\rfloor\}^{\mathbb{N}}$ must satisfy $\sigma^{n}(s)<s$ for all $n \geq 1$. And this condition is sufficient as well. Hence the words $b z_{p, q} b$ and $b\left(z_{p, q} b(b-1)\right)^{\omega}$ above are all greater than their proper suffixes.

Next, we discuss Christoffel words which are less than their tails.

Proposition 2.4. For coprime integers $p$ and $q$, a word $(b-1) z_{p, q} b$ is lexicographically smaller than all its proper suffixes.

COROLlary 2.4.1. $\left((b-1) z_{p, q} b\right)^{\omega}$ is lexicographically smaller than or equal to all suffixes of $b\left(z_{p, q} b(b-1)\right)^{\omega}$.

In the proof of the proposition we will use a fact known as the LyndonSchützenberger theorem. As usual, denote by $\{t\}$ the fractional part of $t$, i.e., $t=\lfloor t\rfloor+\{t\}$.

Theorem 2.5 ([12]). Suppose $y \in A^{*}$ and $x, z \in A^{+}$for some alphabet $A$. Then $x y=y z$ if and only if there exist an integer $e \geq 0$ and words $u, v \in A^{*}$ such that $x=u v, z=v u$, and $y=(u v)^{e} u=u(v u)^{e}$. 
Proof of Proposition 2.4. We may assume $0<p<q$. Recall that if $\alpha=p / q$ then $s_{\alpha, 0}=\left(0 z_{p, q} 1\right)^{\omega}$, and if $0 \leq \varrho<\varrho^{\prime}<1$ then $s_{\alpha, \varrho} \leq s_{\alpha, \varrho^{\prime}}$. Suppose $0 z_{p, q} 1>\sigma^{n}\left(0 z_{p, q} 1\right)=y$ for some $1 \leq n<q$. Letting $0 z_{p, q} 1=y^{\prime} z$ with $|y|=\left|y^{\prime}\right|$ we have $y^{\prime} z>y$. If $y^{\prime}>y$, then one gets $s_{\alpha, 0}=\left(y^{\prime} z\right)^{\omega}>$ $y\left(y^{\prime} z\right)^{\omega}=s_{\alpha,\{\alpha n\}}$. Since $\{\alpha n\}$ is nonzero, this is a contradiction. So we find $y=y^{\prime}$ and hence $0 z_{p, q} 1=y z=x y$ for some $x$. Theorem 2.5 shows that $0 z_{p, q} 1=(u v)^{e+1} u$ for some $u, v \in A^{*}$ and an integer $e \geq 0$. Note that $u$ cannot be the empty word since $0 z_{p, q} 1$ is primitive. Thus one can represent $u$ as $u=0 u^{\prime} 1$, and thus $0 z_{p, q} 1$ as $0 z_{p, q} 1=0 u^{\prime} 1 v(u v)^{e} 0 u^{\prime} 1$. Since $z_{p, q}$ is a palindrome, it follows that $u^{\prime} 1 v(u v)^{e} 0 u^{\prime}=\widetilde{u^{\prime}} 0(\widetilde{v} \widetilde{u})^{e} \widetilde{v} 1 \widetilde{u^{\prime}}$, where $\widetilde{x}$ means the reversal of $x$. We thus get the claimed contradiction.

Definition 2.6. For a rational $\alpha>0, \Delta(\alpha)$ is called a lower selfChristoffel number, and $\Delta(\alpha+):=\lim _{x \rightarrow \alpha+} \Delta(x)$ an upper self-Christoffel number.

As mentioned before, all beta-numbers are algebraic integers. Moreover they are dominant roots of so-called beta-polynomials, and hence Perron numbers [10]. If $d_{\beta}(1)=e_{1} \cdots e_{n}$, then $\beta$ is a zero of the $\beta$-polynomial

$$
x^{n}-\sum_{i=1}^{n} e_{i} x^{n-i},
$$

and if $d_{\beta}(1)=e_{1} \cdots e_{n}\left(e_{n+1} \cdots e_{n+p}\right)^{\omega}$, then $\beta$ is a zero of the $\beta$-polynomial

$$
\left(x^{n+p}-\sum_{i=1}^{n+p} e_{i} x^{n+p-i}\right)-\left(x^{n}-\sum_{i=1}^{n} e_{i} x^{n-i}\right) .
$$

Here we adopt the following widespread abuse of terminology. If $\beta$ is specified in the context, we say " $\beta$-polynomial" instead of "beta-polynomial of $\beta$ ". Self-Christoffel numbers are also zeros of beta-polynomials. We state this as a proposition. For a word $w=a_{0} a_{1} \cdots a_{n-1}$ with $a_{i} \in \mathbb{Z}$, we mean by $\vec{w}$ the vector $\left(a_{0}, \ldots, a_{n-1}\right) \in \mathbb{Z}^{n}$.

Proposition 2.7. Suppose $\alpha=p / q, b=\lceil\alpha\rceil$, and $\operatorname{gcd}(p, q)=1$. Let $\beta=\Delta(\alpha)$ and $\beta^{\prime}=\Delta(\alpha+)$. Then

(a) the $\beta$-polynomial is $x^{q}-\overrightarrow{b z_{p, q} b} \cdot\left(x^{q-1}, x^{q-2}, \ldots, 1\right)$,

(b) the $\beta^{\prime}$-polynomial is $x^{q+1}-\overrightarrow{b z_{p, q} b} \cdot\left(x^{q}, x^{q-1}, \ldots, x\right)-x+1$.

3. Geometry of self-Christoffel numbers. Recall that if $d_{\beta}(1)=$ $e_{1} e_{2} \cdots$, then an equation $1=\sum_{i=1}^{\infty} e_{i} z^{-i}$ has the unique solution $\beta$ in $(1, \infty)$. In fact, one can verify that

$$
1-\sum_{i=1}^{\infty} e_{i} z^{-i}=(1-\beta / z) \sum_{i=0}^{\infty}\left(T_{\beta}^{i} 1\right) z^{-i} .
$$


There has been a geometric study on beta-polynomials. Parry [14] showed that all the conjugates of a beta-number have absolute values less than two. Later this was improved independently by Flatto et al. [7] and by Solomyak [18].

THEOREM 3.1. All the conjugates of a beta-number have absolute values less than $(1+\sqrt{5}) / 2$ and this constant is best possible.

Let $\beta=\Delta(\alpha)$ and $\beta^{\prime}=\Delta(\alpha+)$ be self-Christoffel numbers for some rational $\alpha=p / q, b=\lceil\alpha\rceil$. Then one has $d_{\beta}(1)=b z_{p, q} b$ and $d_{\beta^{\prime}}(1)=$ $b\left(z_{p, q} b(b-1)\right)^{\omega}$. We conclude from Proposition 2.4 that $1-1 / \beta<T_{\beta}^{n} 1 \leq 1$ for $0 \leq n<q$ and $T_{\beta}^{n} 1=0$ for $n \geq q$, and also see that $1-1 / \beta^{\prime}<T_{\beta^{\prime}}^{n} 1 \leq 1$ for all $n \geq 0$.

The next two theorems tell us that Theorem 3.1 can be improved upon for self-Christoffel numbers. In a part of the proof we use similar arguments to those adopted in $[7,18]$.

THEOREM 3.2. Conjugates of an upper self-Christoffel $\beta$ have moduli less than $\left(\beta+\sqrt{\beta^{2}+4 \beta}\right) /(2 \beta)$.

Proof. Suppose $\gamma$ is a conjugate of $\beta$ with $|\gamma|>1$, and let $w=1 / \gamma$. Since $\sum_{i=0}^{\infty}\left(T_{\beta}^{i} 1\right) w^{i}=1+\sum_{i=1}^{\infty}\left(T_{\beta}^{i} 1\right) w^{i}=0$, we have

$$
1+\sum_{i=1}^{\infty} \frac{2 \beta-1}{2 \beta} w^{i}+\sum_{i=1}^{\infty}\left(T_{\beta}^{i} 1-\frac{2 \beta-1}{2 \beta}\right) w^{i}=0,
$$

and therefore

$$
\frac{1}{2 \beta} \frac{|w-2 \beta|}{|w-1|}=\left|1+\sum_{i=1}^{\infty} \frac{2 \beta-1}{2 \beta} w^{i}\right| \leq \sum_{i=1}^{\infty}\left|T_{\beta}^{i} 1-\frac{2 \beta-1}{2 \beta}\right||w|^{i} \leq \frac{1}{2 \beta} \frac{|w|}{1-|w|},
$$

because $1-1 / \beta<T_{\beta}^{n} 1 \leq 1$ for any $n \geq 0$. The curve satisfying $|w| /(1-|w|)$ $=r$ is a circle $x^{2}+y^{2}=r^{2} /(r+1)^{2}$ and the curve satisfying $|w-2 \beta| /(|w-1|)$ $=r$ is a symmetric circle with respect to the $x$-axis having the line segment $[(r-2 \beta) /(r-1),(r+2 \beta) /(r+1)]$ as its diameter. The minimum modulus in the region where the inequality $|w-2 \beta| /|w-1| \leq|w| /(1-|w|)$ holds is attained when for minimal $r>0$ the two circles meet, that is, when

$$
\frac{r-2 \beta}{r-1}=-\frac{r}{r+1} \quad \text { or } \quad r=\frac{\beta+\sqrt{\beta^{2}+4 \beta}}{2} .
$$

So we finally get $|w| \geq\left(-\beta+\sqrt{\beta^{2}+4 \beta}\right) / 2$.

THEOREM 3.3. If $\gamma$ is a conjugate of a lower self-Christoffel $\beta$, then

$$
\frac{2 \beta+1-\sqrt{8 \beta+1}}{2 \beta} \leq|\gamma| \leq \frac{2 \beta+1+\sqrt{8 \beta+1}}{2 \beta} .
$$


Proof. We first assume $|\gamma|>1$ and set $w=1 / \gamma$. Since $\sum_{i=0}^{\infty}\left(T_{\beta}^{i} 1\right) w^{i}=$ $1+\sum_{i=1}^{q-1}\left(T_{\beta}^{i} 1\right) w^{i}=0$, we see that

$$
1+\sum_{i=1}^{q-1} \frac{2 \beta-1}{2 \beta} w^{i}+\sum_{i=1}^{q-1}\left(T_{\beta}^{i} 1-\frac{2 \beta-1}{2 \beta}\right) w^{i}=0,
$$

from which it follows that

$$
\left|1+\sum_{i=1}^{q-1} \frac{2 \beta-1}{2 \beta} w^{i}\right| \leq \sum_{i=1}^{q-1}\left|T_{\beta}^{i} 1-\frac{2 \beta-1}{2 \beta}\right||w|^{i} .
$$

Using $1-1 / \beta<T_{\beta}^{n} 1 \leq 1$ for $1 \leq n \leq q-1$, we derive the inequality as

$$
\begin{gathered}
\left|1+\frac{2 \beta-1}{2 \beta} \cdot \frac{w-w^{q}}{1-w}\right| \leq \frac{1}{2 \beta} \cdot \frac{|w|-|w|^{q}}{1-|w|} \\
\frac{\left|2 \beta\left(1-w^{q}\right)-\left(w-w^{q}\right)\right|}{|1-w|} \leq \frac{|w|-|w|^{q}}{1-|w|}
\end{gathered}
$$

and

$$
\frac{2 \beta\left|1-w^{q}\right|}{|1-w|} \leq \frac{|w|-|w|^{q}}{1-|w|}+\frac{\left|w-w^{q}\right|}{|1-w|} \leq \frac{|w|-|w|^{q}+\left|w-w^{q}\right|}{1-|w|} \leq \frac{2|w|}{1-|w|} .
$$

Consequently, we get

$$
\frac{\beta}{|1-w|} \leq \frac{|w|}{(1-|w|)\left|1-w^{q}\right|} \leq \frac{|w|}{(1-|w|)\left(1-|w|^{q}\right)} \leq \frac{|w|}{(1-|w|)^{2}}
$$

One now finds that $\beta /|w-1|=r$ represents a circle centered at $(1,0)$ with radius $\beta / r$, and $|w| /(1-|w|)^{2}=r$ is a circle that is represented by

$$
|w|=\frac{2 r+1-\sqrt{4 r+1}}{2 r} \text {. }
$$

Then $w$ satisfying $\beta /|w-1| \leq|w| /(1-|w|)^{2}$ with minimum absolute value is attained when

$$
-\frac{2 r+1-\sqrt{4 r+1}}{2 r}=1-\frac{\beta}{r} \quad \text { or } \quad r=\frac{4 \beta-1+\sqrt{8 \beta+1}}{8} .
$$

Hence we have

$$
\frac{1}{|w|} \leq\left(\frac{\beta}{r}-1\right)^{-1}=\frac{r}{\beta-r}=\frac{4 \beta-1+\sqrt{8 \beta+1}}{4 \beta+1-\sqrt{8 \beta+1}}=\frac{2 \beta+1+\sqrt{8 \beta+1}}{2 \beta} .
$$

If $|\gamma|<1$, then one sees that $\gamma^{q-1}+\sum_{i=1}^{q-1}\left(T_{\beta}^{i} 1\right) \gamma^{q-i-1}=0$. We thus have

$$
\begin{aligned}
\left|\gamma^{q-1}+\frac{2 \beta-1}{2 \beta} \cdot \frac{1-\gamma^{q-1}}{1-\gamma}\right| & \leq \frac{1}{2 \beta} \cdot \frac{1-|\gamma|^{q-1}}{1-|\gamma|}, \\
\frac{\left|2 \beta\left(1-\gamma^{q}\right)-\left(1-\gamma^{q-1}\right)\right|}{|1-\gamma|} & \leq \frac{1-|\gamma|^{q-1}}{1-|\gamma|}
\end{aligned}
$$


from which it follows that

$$
\frac{\beta}{|1-\gamma|} \leq \frac{1}{(1-|\gamma|)^{2}}
$$

By a similar argument as before, the minimum absolute value of $\gamma$ satisfying $\beta /|1-\gamma| \leq 1 /(1-|\gamma|)^{2}$ is

$$
|\gamma| \geq \frac{2 \beta+1-\sqrt{8 \beta+1}}{2 \beta}
$$

The reader may have noted that all the bounds given in Theorems 3.2 and 3.3 tend to 1 as $\beta$ tends to infinity. This phenomenon not necessarily occurs for the general beta-numbers.

Example 3.4. Consider $\beta_{b}>1$ for which $d_{\beta_{b}}(1)=b(b 0)^{\omega}$. Then $\beta_{b} \in$ $(b, b+1)$ and the $\beta_{b}$-polynomial is

$$
x^{3}-b x^{2}-(b+1) x+b .
$$

The reasoning that will be developed in the proof of Proposition 3.9 shows that as $b$ tends to infinity the other zeros of the $\beta_{b}$-polynomial tend to those of $x^{2}+\left(1+b^{-1}\right) x-1$, i.e., to

$$
\frac{-\left(1+b^{-1}\right) \pm \sqrt{\left(1+b^{-1}\right)^{2}+4}}{2},
$$

one of whose moduli tends to $(1+\sqrt{5}) / 2$. But $\left(\beta_{b}+\sqrt{\beta_{b}^{2}+4 \beta_{b}}\right) /\left(2 \beta_{b}\right)$ tends to 1 . On the other hand, if $d_{\beta_{b}}(1)=b b 0 b$, then for all sufficiently large $b$ one can find similarly some zero of the $\beta_{b}$-polynomial whose modulus is outside the interval given in Theorem 3.3.

Now the minimal polynomials of self-Christoffel numbers are considered via geometry of numbers. We need some preliminaries.

For a polynomial $g \in \mathbb{Z}[x]$ with leading coefficient $a \neq 0$, the Mahler measure of $g$ is defined by

$$
M(g)=|a| \prod_{g(\alpha)=0} \max \{1,|\alpha|\} .
$$

Clearly, cyclotomic polynomials have Mahler measure 1. In 1933 Lehmer [9] asked whether, for any $h \in \mathbb{Z}[x]$ with $M(h)>1$, there exists an integral polynomial $g$ with $1<M(g)<M(h)$. Despite extensive attempts to answer this question, it has not been settled yet. Among remarkable advances are [3], [19], [6], and the most up-to-date result [20] due to Voutier.

TheOREM 3.5 ([20]). Let $\alpha$ be an algebraic number of degree $n>1$ over $\mathbb{Q}$ with conjugates $\alpha=\alpha_{1}, \alpha_{2}, \ldots, \alpha_{n}$, and suppose $g \in \mathbb{Z}[x]$ is the minimal 
polynomial of $\alpha$. If

$$
\log M(g) \leq \frac{1}{4}\left(\frac{\log \log n}{\log n}\right)^{3}
$$

then $\alpha$ is a root of unity.

Let $f \in \mathbb{Z}[x]$ be the minimal polynomial of a self-Christoffel number $\beta$ (either upper or lower) with $b=\lfloor\beta\rfloor$. If $f=g h$ is a nontrivial factorization over $\mathbb{Q}$ and $g(\beta) \neq 0=h(\beta)$, then Theorems 3.2 and 3.3 together with the above theorem show that $g$ is eventually cyclotomic as $b$ increases. We will take a closer look below into this phenomenon in a more general setting.

A polynomial $R(x)=a_{n} x^{n}+a_{n-1} x^{n-1}+\cdots+a_{0} \in \mathbb{R}[x]$ is reciprocal if $a_{n} a_{n-1} \cdots a_{0}$ is a palindrome as a word. In the case of $\operatorname{deg} R=n$, one sees that $x^{n} R\left(x^{-1}\right)=R(x)$.

Concerning Lehmer's problem there is another result by Smyth, who showed that the problem reduces to the case of reciprocal polynomials.

Theorem $3.6([17])$. Let $p \in \mathbb{Z}[x]$ and let $\theta_{0}=1.32472 \ldots$ be the real root of $x^{3}-x-1=0$. If $M(p)<\theta_{0}$, then $p(x)$ is a reciprocal polynomial.

REMARK 3.7. The above constant is actually a lower self-Christoffel number. One verifies that $\theta_{0}=\Delta(1 / 5)$. Worthy of mention is that $\Delta(1 / 5)$ is the smallest and $\Delta(1 / 4)$ is the second smallest among all Pisot numbers [16]. A Pisot number is an algebraic integer greater than 1 whose conjugates lie inside the unit circle.

Lemma 3.8. Let $R(x)$ be a reciprocal polynomial of degree $q-1$.

(a) If $\gamma$ and $\gamma^{-1}$ are zeros of $x^{q}-R(x)$, then $\gamma^{q+1}=1$.

(b) If $\gamma$ and $\gamma^{-1}$ are zeros of $x^{q+1}-x R(x)-x+1$, then $\gamma^{q-1}=1$.

Proof. We have

$$
\gamma^{q}=R(\gamma)=\gamma^{q-1} R\left(\gamma^{-1}\right)=\gamma^{q-1} \gamma^{-q}=\gamma^{-1} .
$$

This proves part (a). Similarly we have

$$
\gamma=\gamma^{q+1}-\gamma R(\gamma)+1=\gamma^{q+1} \gamma^{-1}=\gamma^{q},
$$

giving $\gamma^{q-1}=1$.

Let $x_{1}, \ldots, x_{n}$ be $n$ indeterminates. The elementary symmetric functions with respect to $x_{1}, \ldots, x_{n}$ are the multivariate polynomials defined by

$$
E_{1}=\sum_{i=1}^{n} x_{i}, \quad E_{2}=\sum_{1 \leq i<j \leq n} x_{i} x_{j}, \quad \ldots, \quad E_{n}=\prod_{i=1}^{n} x_{i} .
$$

It is well known that the Jacobian determinant of $E_{1}, \ldots, E_{n}$ equals the 
Vandermonde determinant of $x_{1}, \ldots, x_{n}$, i.e.,

$$
J\left(E_{1}, \ldots, E_{n}\right)=\left|\frac{\partial\left(E_{1}, \ldots, E_{n}\right)}{\partial\left(x_{1}, \ldots, x_{n}\right)}\right|=\prod_{1 \leq i<j \leq n}\left(x_{i}-x_{j}\right) .
$$

Let $f(x)=\sum_{i=0}^{n} a_{i} x^{i}, g(x)=\sum_{i=0}^{n} b_{i} x^{i}$ be of the same degree in $\mathbb{R}[x]$. If $f(x)$ is separable, then the inverse function theorem tells us that if every pair $\left(a_{i}, b_{i}\right)$ is such that the two numbers are sufficiently close to each other then there is a similar relation between the zeros of $f$ and $g$. But quantifying how close to each other both zeros are, is a hard task in general. The main difficulty lies in insolvability of quintic and higher degree polynomials. It is well known that the implicit function theorem is a special case of the inverse function theorem. In fact, they are equivalent.

Proposition 3.9. Suppose $c_{q}(x)$ is a (product of) cyclotomic polynomial(s) with $\operatorname{deg} c_{q}=q-1$ and it has no multiple roots. Let $R(x)=$ $a_{q-1} x^{q-1}+\cdots+a_{0} \in \mathbb{Z}[x]$ be reciprocal with $\operatorname{deg} R \leq q-1$.

(a) If $\operatorname{gcd}\left(c_{q}(x), x^{q+1}-1\right)=1$ then $f_{1}(x)=x^{q}+R(x)-b c_{q}(x)$ is irreducible for all sufficiently large $b$.

(b) If $\operatorname{gcd}\left(c_{q}(x), x^{q-1}-1\right)=1$ then $f_{2}(x)=x^{q+1}+x\left(R(x)-b c_{q}(x)\right)-x+1$ is irreducible for all sufficiently large $b$.

Proof. Considering $f_{1}(x) / b$ (resp. $f_{2}(x) / b$ ), we see that the inverse function theorem shows that each of the $q-1$ (resp. q) zeros of $f_{1}(x)$ (resp. $f_{2}(x)$ ) other than the dominant zero approaches the corresponding zero of $c_{q}(x)$ (resp. $\left.x c_{q}(x)\right)$ as $b$ increases. On the other hand, the final real zero $\beta_{1}$ (resp. $\beta_{2}$ ) tends to infinity since the trace of $\beta_{1}$ (resp. $\beta_{2}$ ) tends to infinity as $b$ increases.

Suppose that $f_{i}=g_{i} h_{i}, i=1,2$, with $g_{i}\left(\beta_{i}\right) \neq 0=h_{i}\left(\beta_{i}\right)$. Then Theorem 3.6 implies that $g_{i}$ is eventually reciprocal as $b$ increases. By Lemma 3.8, we get $\operatorname{gcd}\left(g_{1}(x), x^{q+1}-1\right) \neq 1$ and $\operatorname{gcd}\left(g_{2}(x), x^{q-1}-1\right) \neq 1$.

COROLlary 3.9.1. Let $p / q$ be a fixed rational with $0<p \leq q$ and $\operatorname{gcd}(p, q)=1$. For a positive integer $b$, let $\alpha=b-1+p / q, \beta=\Delta(\alpha)$ and $\beta^{\prime}=\Delta(\alpha+)$. Then for all sufficiently large $b$,

(a) the $\beta$-polynomial $x^{q}-\overrightarrow{b z_{p, q} b} \cdot\left(x^{q-1}, x^{q-2}, \ldots, 1\right)$ is irreducible,

(b) the $\beta^{\prime}$-polynomial $x^{q+1}-\overrightarrow{b z_{p, q} b} \cdot\left(x^{q}, x^{q-1}, \ldots, x\right)-x+1$ is irreducible.

According to the proof of Proposition 3.9, all the conjugates of selfChristoffel numbers approach the roots of unity or zero. On the complex plane $\mathbb{C}$, Figure 1 represents conjugates of lower self-Christoffel numbers $\Delta(11 / 7)$ and $\Delta(704 / 7)$, and Figure 2 represents conjugates of upper selfChristoffel numbers $\Delta(11 / 7+)$ and $\Delta(704 / 7+)$. The reader can verify, using some symbolic calculation package, that $\operatorname{deg}(\Delta(11 / 7))=\operatorname{deg}(\Delta(704 / 7))=7$ 

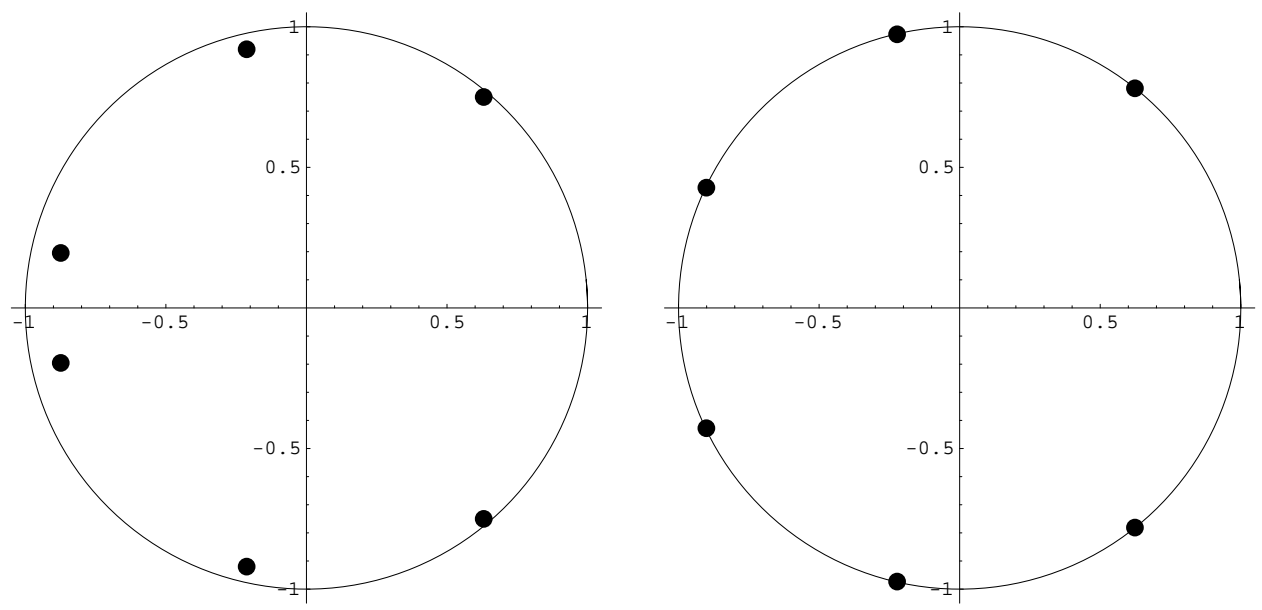

Fig. 1. Conjugates of $\Delta(11 / 7)$ (left) and $\Delta(704 / 7)$ (right)
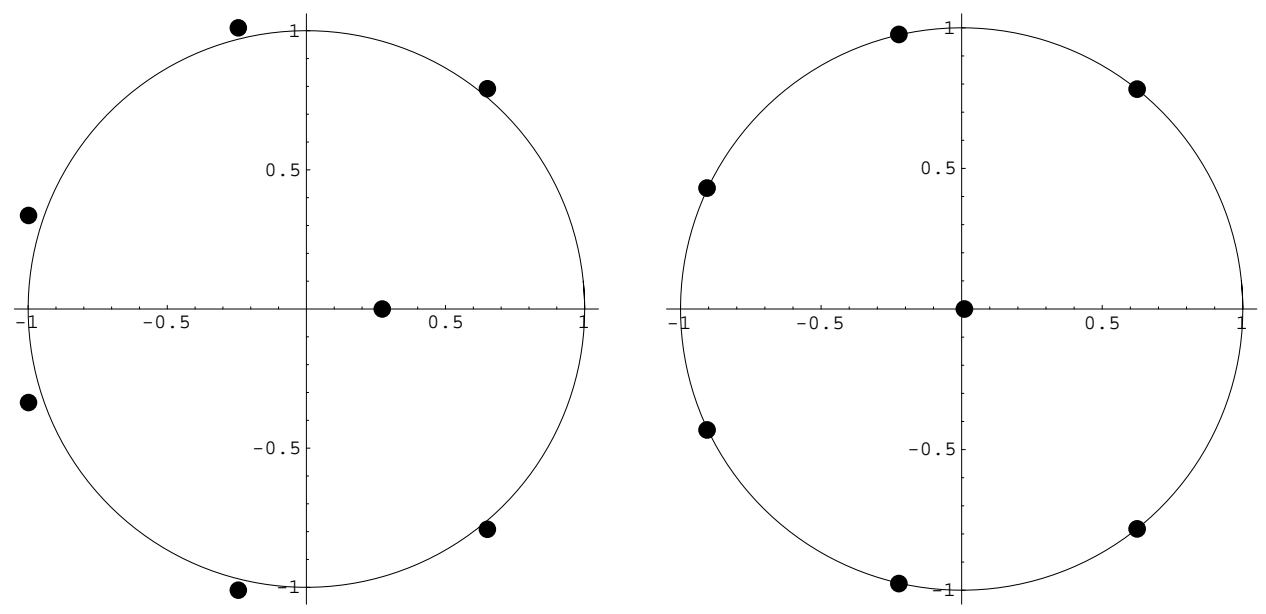

Fig. 2. Conjugates of $\Delta(11 / 7+)$ (left) and $\Delta(704 / 7+)$ (right)

and that $\operatorname{deg}(\Delta(11 / 7+))=\operatorname{deg}(\Delta(704 / 7+))=8$. In other words, their betapolynomials are all irreducible over $\mathbb{Q}$. In both cases, each nonreal conjugate of $\Delta(704 / 7)$ and $\Delta(704 / 7+)$ seems to be very close to some seventh root of unity.

We note the following fact. Its proof is a straightforward computation from equation (1).

LEMMA 3.10 .

(a) If $d_{\beta}(1)=e_{1} \cdots e_{n}$, then the $\beta$-polynomial factors over $\mathbb{Q}(\beta)$ into

$$
x^{n}-\sum_{i=1}^{n} e_{i} x^{n-i}=(x-\beta) \sum_{i=0}^{n-1}\left(T_{\beta}^{i} 1\right) x^{n-i-1} .
$$


(b) If $d_{\beta}(1)=e_{1} \cdots e_{n}\left(e_{n+1} \cdots e_{n+p}\right)^{\omega}$, then the $\beta$-polynomial factors over $\mathbb{Q}(\beta)$ into

$$
\begin{aligned}
\left(x^{n+p}-\sum_{i=1}^{n+p}\right. & \left.e_{i} x^{n+p-i}\right)-\left(x^{n}-\sum_{i=1}^{n} e_{i} x^{n-i}\right) \\
& =(x-\beta)\left(\sum_{i=0}^{n+p-1}\left(T_{\beta}^{i} 1\right) x^{n+p-i-1}-\sum_{i=0}^{n-1}\left(T_{\beta}^{i} 1\right) x^{n-i-1}\right) .
\end{aligned}
$$

Let $\alpha=b-1+p / q, 0<p \leq q, \operatorname{gcd}(p, q)=1$ and $\beta=\Delta(\alpha+)$. As for upper self-Christoffel numbers, the above lemma reads

$$
\begin{array}{r}
x^{q+1}-\overrightarrow{b z_{p, q} b} \cdot\left(x^{q}, x^{q-1}, \ldots, x\right)-x+1=(x-\beta)\left(\sum_{i=0}^{q}\left(T_{\beta}^{i} 1\right) x^{q-i}-1\right) \\
=(x-\beta)\left(\left(T_{\beta}^{0} 1\right) x^{q}+\left(T_{\beta}^{1} 1\right) x^{q-1}+\cdots+\left(T_{\beta}^{q-1} 1\right) x-\frac{1}{\beta}\right) .
\end{array}
$$

As a result, one can readily see that the $\beta$-polynomial has a positive zero in $(0,1)$. We state this more precisely.

Proposition 3.11. Let $p, q, b$ and $\alpha$ be as in Corollary 3.9 .1 and let $\beta=\Delta(\alpha+)$. Then the $\beta$-polynomial has a real zero $\beta_{q} \in(0,1 / \beta)$.

Computation shows that many of lower self-Christoffel numbers are in fact Pisot numbers. But the proposition implies that this is not the case for upper self-Christoffel numbers. Indeed, if $\beta_{q}$ were a zero of the minimal polynomial of an upper self-Christoffel number $\beta$ and if $\beta$ were a Pisot number, then the norm of $\beta$ could not be an integer.

Proof of Proposition 3.11. First we note that $10^{\omega}$ and $0 b\left(z_{p, q} b(b-1)\right)^{\omega}$ in $S_{\beta}$ represent the same real number $1 / \beta$, which we denote by $10^{\omega} \equiv_{\beta}$ $0 b\left(z_{p, q} b(b-1)\right)^{\omega}$. Set

$$
f(x):=\sum_{i=0}^{q-1}\left(T_{\beta}^{i} 1\right) x^{q-i}=\left(T_{\beta}^{0} 1\right) x^{q}+\left(T_{\beta}^{1} 1\right) x^{q-1}+\cdots+\left(T_{\beta}^{q-1} 1\right) x
$$

and let $z_{p, q}=z_{1} \cdots z_{q-2}$. Now the $\beta$-expansion of $\left(T_{\beta}^{q-i} 1\right) / \beta^{i}$ is

$$
\begin{cases}0\left(b(b-1) z_{1} \cdots z_{q-2}\right)^{\omega} & \text { for } i=1 \\ 0^{i}\left(z_{q-i} \cdots z_{q-2} b(b-1) z_{1} \cdots z_{q-i-1}\right)^{\omega} & \text { for } 2 \leq i \leq q-2 \\ 0^{q-1}\left(z_{1} \cdots z_{q-2} b(b-1)\right)^{\omega} & \text { for } i=q-1\end{cases}
$$

Then the first letters after $0^{i}(i=1, \ldots, q-1)$ constitute a word

$$
b z_{q-2} z_{q-3} \cdots z_{1}=b z_{p, q}
$$

since $z_{p, q}$ is a palindrome. Finally, the $\beta$-expansion of $\left(T_{\beta}^{0} 1\right) / \beta^{q}$ is given by $0^{q-1} 10^{\omega} \equiv{ }_{\beta} 0^{q} b\left(z_{p, q} b(b-1)\right)^{\omega}$. Gathering the above, one finds that the 
$\beta$-expansion of $f\left(\beta^{-1}\right)$ satisfies

$$
d_{\beta}\left(f\left(\beta^{-1}\right)\right)>0 b z_{p, q} b\left(z_{p, q} b(b-1)\right)^{\omega}>0 b z_{p, q} b\left((b-1) z_{p, q} b\right)^{\omega} \equiv \equiv_{\beta} 10^{\omega},
$$

where the last inequality follows from Proposition 2.4. Thus we get $f\left(\beta^{-1}\right)$ $>1 / \beta$.

\section{Beta-numbers whose conjugates lie near the roots of unity.} Let us fix a Christoffel word over an alphabet $\{b-1, b\}$. If $f_{1}$ and $f_{2}$ are the beta-polynomials of the corresponding lower and upper self-Christoffel numbers respectively, then it follows from Proposition 3.9 that $f_{1}(x) /(x-\beta)$ tends to $c_{q}(x)$ and $f_{2}(x) /(x-\beta)$ to $x c_{q}(x)$ as $b$ increases. In what follows, we show that some converses of these properties are also true. In other words, if all conjugates of a beta-number $\beta$ are "sufficiently" close to the roots of unity (or to zero in the case of upper self-Christoffel numbers), then $\beta$ is indeed a self-Christoffel number. Here "sufficiently" means that inequalities (2) and (4) hold. Now the inverse function theorem justifies the title of this section. While the beta-polynomial of a lower (resp. upper) self-Christoffel number with degree $q$ is far from $x^{q}-1$ (resp. $\left.x^{q}-x\right)$, all its conjugates are close to some zeros of $x^{q}-1$ (resp. $\left.x^{q}-x\right)$. Furthermore this property distinguishes self-Christoffel numbers from the other beta-numbers.

THEOREM 4.1. Suppose $\beta$ is a simple beta-number with $d_{\beta}(1)=e_{1} \cdots e_{q}$ and $\beta_{1}, \ldots, \beta_{q-1}$ are the other zeros of the $\beta$-polynomial. If, for all $j=$ $1, \ldots, q-1$,

$$
1-1 / \beta<\left|E_{j}\left(\beta_{1}, \ldots, \beta_{q-1}\right)\right|<1,
$$

then $\beta$ is a lower self-Christoffel number.

Proof. By Lemma 3.10, $\beta_{1}, \ldots, \beta_{q-1}$ are zeros of $\sum_{j=0}^{q-1}\left(T_{\beta}^{j} 1\right) x^{q-j-1}$. Since $T_{\beta}^{j} 1=(-1)^{j} E_{j}\left(\beta_{1}, \ldots, \beta_{q-1}\right)$ for $j=1, \ldots, q-1$, one has

$$
1-1 / \beta<T_{\beta}^{j} 1<1 \quad \text { for } j=1, \ldots, q-1 .
$$

Hence $\operatorname{alph}\left(d_{\beta}(1)\right)=\left\{e_{1}-1, e_{1}\right\}$. Let $a=e_{1}-1$ and $b=e_{1}$. Since $d_{\beta}(1-1 / \beta)=a e_{2} \cdots e_{q}$, one readily notes from inequality (3) that $e_{q}$ is equal to $b$.

We claim that the finite words $d_{\beta}(1)$ and $d_{\beta}(1-1 / \beta)$ are balanced. Otherwise, if $d_{\beta}(1-1 / \beta)$ is unbalanced, then Proposition 2.1 gives us a palindrome $w$ for which both $a w a$ and $b w b$ are factors of $d_{\beta}(1-1 / \beta)$. Whether awa is a prefix of $d_{\beta}(1-1 / \beta)$ or not, inequality (3) now guarantees that

$$
a e_{2} \cdots e_{n+2} \leq a w a<b w b \leq b e_{2} \cdots e_{n+2},
$$


where $n$ is the length of $w$. But this yields a contradiction $e_{2} \cdots e_{n+2}<$ $e_{2} \cdots e_{n+2}$, and so $d_{\beta}(1-1 / \beta)$ is balanced. A similar argument shows that $d_{\beta}(1)$ is balanced. Now Proposition 2.2 proves that $e_{2} \cdots e_{q}$ is a prefix of some characteristic word.

Suppose $a e_{2} \cdots e_{q}$ is a prefix of a lower mechanical word $s_{\alpha_{0}, 0}$ and put

$$
\delta=\min \left\{\left\{\alpha_{0} n\right\} / n \mid 1 \leq n \leq q\right\} .
$$

Then $\alpha=\alpha_{0}-\delta$ is equal to some rational $p / r$ with $\operatorname{gcd}(p, r)=1$ and $r \leq q$, and $a e_{2} \cdots e_{q}$ is also a prefix of $s_{\alpha, 0}$. Suppose $r<q$. Then $a e_{2} \cdots e_{q}=$ $\left(a z_{p, r} b\right)^{e} u$ for some $e \geq 1$ and $u$ is a prefix of $a z_{p, r} b$. For $u$ nonempty, we thus have $a e_{2} \cdots e_{q}>u$. If $u$ is the empty word, then $e \geq 2$ and so $\left(a z_{p, r} b\right)^{e}>a z_{p, r} b$. Either case contradicts (3).

As for upper self-Christoffel numbers, we need to consider the length of a "preperiod" as follows.

TheOREM 4.2. Suppose $\beta$ is a beta-number with

$$
d_{\beta}(1)=e_{1} \cdots e_{n}\left(e_{n+1} \cdots e_{q+1}\right)^{\omega}
$$

and $\beta_{1}, \ldots, \beta_{q}$ are the other zeros of the $\beta$-polynomial. If, for all $j=$ $1, \ldots, q-1$,

$$
1-1 / \beta \leq\left|E_{j}\left(\beta_{1}, \ldots, \beta_{q}\right)\right|<1 \quad \text { and } \quad\left|E_{q}\left(\beta_{1}, \ldots, \beta_{q}\right)\right| \leq 1 / \beta,
$$

then $\beta$ is an upper self-Christoffel number.

Note that we do not assume $n=1$.

Proof. We use a reasoning similar to the proof of Theorem 4.1.

All of $\beta_{1}, \ldots, \beta_{q}$ are zeros of

$$
\begin{aligned}
& \left(\sum_{i=0}^{q}\left(T_{\beta}^{i} 1\right) x^{q-i}-\sum_{i=0}^{n-1}\left(T_{\beta}^{i} 1\right) x^{n-i-1}\right)=T_{\beta}^{0} 1 \cdot x^{q}+\cdots+T_{\beta}^{q-n} 1 \cdot x^{n} \\
& \quad+\left(T_{\beta}^{q-n+1} 1-T_{\beta}^{0} 1\right) \cdot x^{n-1}+\cdots+\left(T_{\beta}^{q-1} 1-T_{\beta}^{n-2} 1\right) \cdot x+\left(T_{\beta}^{q} 1-T_{\beta}^{n-1} 1\right) .
\end{aligned}
$$

First we prove $n=1$. Suppose $n>1$. Then $1-1 / \beta \leq T_{\beta}^{q-n+1} 1-T_{\beta}^{0} 1<1$ or $T_{\beta}^{q-n+1} 1 \geq 2-1 / \beta$, a contradiction. So we have

$$
1-1 / \beta \leq T_{\beta}^{j} 1<1 \quad \text { for } j=1, \ldots, q \text {. }
$$

So $\operatorname{alph}\left(d_{\beta}(1)\right)=\left\{e_{1}-1, e_{1}\right\}$. Let $a=e_{1}-1$ and $b=e_{1}$. The same argument as before shows that the word $d_{\beta}(1-1 / \beta)=a\left(e_{2} \cdots e_{q+1}\right)^{\omega}$ is balanced. Note that the $\beta$-expansion of $T_{\beta}^{q} 1$ is $e_{q+1}\left(e_{2} \cdots e_{q+1}\right)^{\omega}$. If $e_{q+1}=b$, then we get $T_{\beta}^{q} 1=1$, a contradiction. So the word $d_{\beta}(1-1 / \beta)=a\left(e_{2} \cdots e_{q} a\right)^{\omega}=$ $\left(a e_{2} \cdots e_{q}\right)^{\omega}$ is purely periodic. We conclude from Proposition 2.2 that $\beta$ is an upper self-Christoffel number. 


\section{References}

[1] A. Bertrand-Mathis, Développement en base $\theta$; répartition modulo un de la suite $\left(x \theta^{n}\right)_{n \geq 0}$; langages codés et $\theta$-shift, Bull. Soc. Math. France 114 (1986), 271-323.

[2] F. Blanchard, $\beta$-expansions and symbolic dynamics, Theoret. Comput. Sci. 65 (1989), 131-141.

[3] P. E. Blanksby and H. L. Montgomery, Algebraic integers near the unit circle, Acta Arith. 18 (1971), 355-369.

[4] D. P. Chi and D.Y. Kwon, Sturmian words, $\beta$-shifts, and transcendence, Theoret. Comput. Sci. 321 (2004), 395-404.

[5] E. M. Coven and G. A. Hedlund, Sequences with minimal block growth, Math. Systems Theory 7 (1973), 138-153.

[6] E. Dobrowolski, On a question of Lehmer and the number of irreducible factors of a polynomial, Acta Arith. 34 (1979), 391-401.

[7] L. Flatto, J. C. Lagarias and B. Poonen, The zeta function of the beta transformation, Ergodic Theory Dynam. Systems 14 (1994), 237-266.

[8] D.Y. Kwon, A devil's staircase from rotations and irrationality measures for Liouville numbers, preprint, 2004, available at http://math.yonsei.ac.kr/doyong/paper/ devil_pre.pdf.

[9] D. H. Lehmer, Factorization of certain cyclotomic functions, Ann. of Math. (2) 34 (1933), 461-479.

[10] D. A. Lind, The entropies of topological Markov shifts and a related class of algebraic integers, Ergodic Theory Dynam. Systems 4 (1984), 283-300.

[11] M. Lothaire, Algebraic Combinatorics on Words, Cambridge Univ. Press, 2002.

[12] R. C. Lyndon and M. P. Schützenberger, The equation $a^{M}=b^{N} c^{P}$ in a free group, Michigan Math. J. 9 (1962), 289-298.

[13] M. Morse and G. A. Hedlund, Symbolic dynamics II. Sturmian trajectories, Amer. J. Math. 62 (1940), 1-42.

[14] W. Parry, On the $\beta$-expansions of real numbers, Acta Math. Acad. Sci. Hungar. 11 (1960), 401-416.

[15] A. Rényi, Representations for real numbers and their ergodic properties, ibid. 8 (1957), 477-493.

[16] C. L. Siegel, Algebraic integers whose conjugates lie in the unit circle, Duke Math. J. 11 (1944), 597-602.

[17] C. J. Smyth, On the product of the conjugates outside the unit circle of an algebraic integer, Bull. London Math. Soc. 3 (1971), 169-175.

[18] B. Solomyak, Conjugates of beta-numbers and the zero-free domain for a class of analytic functions, Proc. London Math. Soc. (3) 68 (1994), 477-498.

[19] C. L. Stewart, Algebraic integers whose conjugates lie near the unit circle, Bull. Soc. Math. France 106 (1978), 169-176.

[20] P. Voutier, An effective lower bound for the height of algebraic numbers, Acta Arith. 74 (1996), 81-95.

Department of Mathematics

Yonsei University

134 Shinchon-dong, Seodaemun-gu

Seoul 120-749, Republic of Korea

E-mail: doyong@yonsei.ac.kr 